\title{
A complex information quality for complex mass function in evidence theory
}

This paper was downloaded from TechRxiv (https://www.techrxiv.org).

\section{LICENSE}

$\mathrm{CCO}$

SUBMISSION DATE / POSTED DATE

$10-04-2020 / 11-04-2020$

\section{CITATION}

Xiao, Fuyuan (2020): A complex information quality for complex mass function in evidence theory. TechRxiv. Preprint. https://doi.org/10.36227/techrxiv.12109158.v1

DOI

10.36227/techrxiv.12109158.v1 


\title{
A complex information quality for complex mass function in evidence theory
}

\author{
Fuyuan Xiao, Member, IEEE
}

\begin{abstract}
In this paper, a complex information quality measure is proposed for complex mass functions.

Index Terms-Generalized Dempster-Shafer evidence theory, Complex evidence theory, Complex mass function, Complex basic belief assignments, Complex information quality.
\end{abstract}

\section{INTRODUCTION}

A recent work in terms of the generalization of DempsterShafer evidence (GDSE) theory is presented where a new concept of complex belief function is defined based on the complex numbers [1, 2]. The GDSE theory is capable of giving expression to the data fluctuations at a given time phase in the course of execution. Moreover, it has the ability to handle uncertainty and imprecision when the data occur concurrently accompanied by variations against to data's phase or periodicity. In particular, when the complex basic belief assignments are degenerated from complex numbers to real numbers, the GDSE theory will degrade into the DSE theory with the condition that the conflict coefficient is less than one. Therefore, the GDSE theory can provide a more promising framework to model and cope with uncertain information. In this paper, inspired by Yager and Petry's work [3], a complex information quality measure is proposed for complex mass functions in GDSE theory.

\section{COMPlEX MASS FUNCTION}

A generalization of Dempster-Shafer evidence (GDSE) theory is presented recently, in which a new concept of complex belief function is defined based on the complex numbers [1, 2].

Let $\Omega$ be a set of mutually exclusive and collective nonempty events, defined by

$$
\Omega=\left\{e_{1}, e_{2}, \ldots, e_{i}, \ldots, e_{n}\right\},
$$

where $\Omega$ represents a frame of discernment.

The power set of $\Omega$ is denoted by $2^{\Omega}$, in which

$$
\begin{array}{r}
2^{\Omega}=\left\{\emptyset,\left\{e_{1}\right\},\left\{e_{2}\right\}, \ldots,\left\{e_{n}\right\},\left\{e_{1}, e_{2}\right\}, \ldots,\left\{e_{1},\right.\right. \\
\left.\left.e_{2}, \ldots, e_{i}\right\}, \ldots, \Omega\right\},
\end{array}
$$

and $\emptyset$ is an empty set.

\section{Definition 1 (Complex mass function)}

Corresponding author: Fuyuan Xiao.

F. Xiao is an Associate Professor at the School of Computer and Information Science, Southwest University, No.2 Tiansheng Road, BeiBei District, Chongqing, 400715, China (e-mail: xiaofuyuan@swu.edu.cn).
A complex mass function $\mathrm{M}$ in the frame of discernment $\Omega$ is modeled as a complex number, which is represented as a mapping from $2^{\Omega}$ to $\mathbb{C}$, defined by

$$
\mathrm{M}: \quad 2^{\Omega} \rightarrow \mathbb{C},
$$

satisfying the following conditions,

$$
\begin{aligned}
\mathbb{M}(\emptyset) & =0, \\
\sum_{A \in 2^{\Omega}} \mathbb{M}(A) & =\mathbf{m}(A) e^{i \theta(A)}, \quad A \in 2^{\Omega}
\end{aligned}
$$

where $i=\sqrt{-1} ; \mathbf{m}(A) \in[0,1]$ representing the magnitude of the complex mass function $\mathbb{M}(A) ; \theta(A) \in[-\pi, \pi]$ denoting a phase term.

In Eq. (4), $\mathrm{M}(A)$ can also be expressed in the "rectangular" form or "Cartesian" form, denoted by

$$
\mathbb{M}(A)=x+y i, \quad A \in 2^{\Omega}
$$

with

$$
\sqrt{x^{2}+y^{2}} \in[0,1]
$$

By using the Euler's relation, the magnitude and phase of the complex mass function $\mathrm{M}(A)$ can be expressed as

$$
\mathbf{m}(A)=\sqrt{x^{2}+y^{2}}, \text { and } \theta(A)=\arctan \left(\frac{y}{x}\right),
$$

where $x=\mathbf{m}(A) \cos (\theta(A))$ and $y=\mathbf{m}(A) \sin (\theta(A))$.

The square of the absolute value for $\mathrm{M}(A)$ is defined by

$$
|\mathrm{M}(A)|^{2}=\mathbb{M}(A) \overline{\mathrm{M}}(A)=x^{2}+y^{2},
$$

where $\overline{\mathrm{M}}(A)$ is the complex conjugate of $\mathrm{M}(A)$, such that $\overline{\mathrm{M}}(A)=x-y i$.

These relationships can be then obtained as

$$
\mathbf{m}(A)=|\mathbb{M}(A)|, \text { and } \theta(A)=\angle \mathbb{M}(A),
$$

where if $\operatorname{M}(A)$ is a real number (i.e., $y=0$ ), then $\mathbf{m}(A)=$ $|x|$.

If $|\mathrm{M}(A)|\left(A \in 2^{\Omega}\right)$ is greater than zero, $A$ is called a focal element of the complex mass function. The value of $|\mathrm{M}(A)|$ represents how strongly the evidence supports $A$.

The complex mass function $\mathbb{M}$ modeled as a complex number in the generalized Dempster-Shafer (GDS) evidence theory can also be called a complex basic belief assignment (CBBA). When $\mathrm{M}(A)$ degrades into a real number, a CBBA will degrades into a BBA. 
III. A COMPLEX INFORMATION QUALITY FOR CBBAS

Definition 2 (Complex information quality)

The complex information quality for CBBAs $\mathrm{M}$, denoted as $\mathrm{IQ}\left(\overrightarrow{\mathrm{IM}}_{1}, \overrightarrow{\mathrm{M}}_{2}\right)$ is defined by

$$
\mathrm{IQ}(\mathrm{IM})=\|\mathrm{M}\|^{2}=\frac{\mathrm{M}(A)}{2^{|A|}-1} \cdot \frac{\overline{\mathrm{M}}(A)}{2^{|A|}-1} .
$$

The complex information quality is a generalization of Yager and Petry's work [3].

\section{CONCLUSiOns}

In this paper, a complex information quality measure was proposed for complex mass functions in a more general framework of complex plane space.

\section{CONFLICT OF INTEREST}

The author states that there are no conflicts of interest.

\section{ACKNOWLEDGMENTS}

This research is supported by the Fundamental Research Funds for the Central Universities (No. XDJK2019C085) and Chongqing Overseas Scholars Innovation Program (No. cx2018077).

\section{REFERENCES}

[1] F. Xiao, "Generalization of Dempster-Shafer theory: A complex mass function," Applied Intelligence, pp. DOI: 10.1007/s10 489-019-01 617-y, 2019.

[2] — , "Generalized belief function in complex evidence theory," Journal of Intelligent \& Fuzzy Systems, pp. DOI: 10.3233/JIFS-179 589, 2020.

[3] R. R. Yager and F. Petry, "An intelligent quality-based approach to fusing multi-source probabilistic information," Information Fusion, vol. 31, pp. 127-136, 2016. 